\title{
Mini-commentary on BJOG-20-0939.R2
}

\author{
Miho Iida ${ }^{1}$ and Mamoru Tanaka ${ }^{2}$ \\ ${ }^{1}$ Keio University School of Medicine Graduate School of Medicine \\ ${ }^{2}$ Keio University School of Medicine
}

July 21, 2020

With no end in sight to the convergence of COVID-19, countries are struggling with strategies to halt the "second wave" and mitigate economic decline. Estimated to account for around half of the infections, asymptomatic transmission of SARS-CoV-2 has been hampering the containment of the virus. A positive case rate of $10 \%$ was reported by Prabhu et al. among 625 pregnant women who were universally screened for SARS-CoV-2 on the day of admission for delivery at 3 institutions in New York City, of which $80 \%$ were asymptomatic at the time of testing including pre- and post-symptomatic patients. As evidence shows that virus sheds before symptoms appear and even after their cessation, these populations may have increased the chances of COVID-19 outbreak in the hospitals. Utilization of testing results for isolation practices was not mentioned in the report, possibly given the long turnaround time for the testing platforms at the time.

While the risk of nosocomial transmission is affected by clinical settings, the intimate and prolonged nature of childbirth elevates the risk of cross-infection between midwives and women. The role of nosocomial transmission has been increasingly recognized, and its severity risk may be greater than those of communityacquired infections. A recent report has suggested facilities to consider testing pregnant women for SARSCoV-2 at the time of admission (Rasmussen SA, et al. JAMA. 2020). International Confederation of Midwives has also called for governments to prioritize testing for all pregnant women and their care providers. Identification of infectious women prior to delivery could contribute to prevention of further transmission to patients and healthcare workers. Importance should also be emphasized on evaluating contact history due to the nature of false-negative PCR results (Woloshin S, et al. NEJM. 2020).

Another significance of performing testing for SARS-CoV-2 on pregnant women is for the adequate medical management of the women and the fetuses. While outcome for mothers and neonates seems generally favorable, data suggest that pregnancy can be associated with increased risk for severity, including intensive care unit admission and receipt of mechanical ventilation (Ellington S, et al., MMWR Morb Mortal Wkly Rep 2020;69:769-775). Furthermore, a recent article has raised concerns over transplacental transmission of SARS-CoV-2 to the fetus (Vivanti AJ, et al. Nat Commun. 2020;11:3572). Collection of longitudinal data is crucial to understand the effects of SARS-CoV-2 infection on maternal and neonatal outcomes. Results of large-scale prospective cohort studies, such as INTERCOVID study, are expected to add high-quality evidence on the effects of COVID-19 in pregnancy on the health of the mothers, fetuses, and newborns.

Screening a maternity population under a pandemic can be a way to provide a glimpse of the distribution of the population, since capacity constraints still impede widespread testing in many countries. Recent development of faster diagnostic testing could bring improvement, but test sensitivity will remain a challenge.

Fundamental preventive measures and clinical management should be continued; that is, hygiene and social distancing practices for women themselves, and careful evaluation of each mother and fetus for care providers. 